\title{
Noncommutative Wormholes in $f(R)$ Gravity with Lorentzian Distribution
}

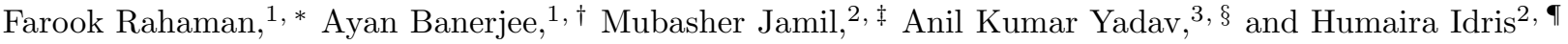 \\ ${ }^{1}$ Department of Mathematics, Jadavpur University, Kolkata 700 032, West Bengal, India \\ ${ }^{2}$ School of Natural Sciences, National University of Sciences and Technology (NUST), H-12, Islamabad, Pakistan \\ ${ }^{3}$ Department of Physics, Anand Engineering College, Keetham, Agra 282 007, India
}

\begin{abstract}
In this paper, we derive some new exact solutions of static wormholes in $f(R)$ gravity supported by the matter possesses Lorentizian density distribution of a particle-like gravitational source. We derive the wormhole's solutions in two possible schemes for a given Lorentzian distribution: assuming an astrophysically viable $F(R)$ function such as a power-law form and discuss several solutions corresponding to different values of the exponent ( here $F=\frac{d f}{d R}$ ). In the second scheme, we consider particular form of two shape functions and have reconstructed $f(R)$ in both cases. We have discussed all the solutions with graphical point of view.
\end{abstract}

Keywords: Wormhole; non-commutative geometry; Modified gravity.

PACS numbers: 04.50.-h, 04.50.Kd, 04.20.Jb

\section{INTRODUCTION}

The geometry of a static and stationary spherically symmetric wormhole consists of a two-mouthed tunnel (referred as a tube, throat, or handle in the literature). This tube like structure is a multiply connected spacetime which can join two asymptotically flat regions of the same spacetime or two different spacetimes. The possibility of two way travel in time becomes theoretically possible when the two regions belong to the same spacetime, such phenomenon is time machine. The wormhole spacetime has been investigated in the literature in various schemes such as a non-static axially symmetric wormhole [1], wormholes with cylindrical symmetry [2], wormholes supported by a cosmological constant [3], thin-shell wormholes [4], and electrically charged static wormholes [5] (For a review see [6]). Morris and Thorne 7] suggested the idea of a traversable wormhole suitable for travel by humans for interstellar journey and beyond. Later on wormholes were investigated in the cosmological context and theorized to be enlarged through a mechanism similar to cosmological inflation [8]. Wormholes present esoteric properties such as a violation of the Hawking chronology protection conjecture and give faster-than-light scenarios, breakdown of causality [9]. The matter energy supporting the exotic geometry violates the standard energy conditions and hence termed 'exotic'. However this exotic behavior can be avoided by studying wormholes in extended theories of gravity such as $f(R)$ gravity and threading wormholes with normal matter. The violation of energy conditions can be avoided on account of curvature effects arising near the wormhole's throat.

In an earlier work by Lobo et al [1], the authors investigated the geometry and stability of stationary and static wormholes in $f(R)$ gravity. They obtained the wormhole's solutions by assuming various forms of equations of state and viable shape functions. They showed that in their model, the energy conditions are satisfied in the desired range of radial coordinate. We here perform a similar analysis of [11] however, taking into account a Lorentizian density distribution of a point gravitational source [12]. We derive the wormhole's solutions in two possible schemes for a given Lorentzian distribution: assuming an astrophysically viable $F(R)$ function such as a power-law form $: F(R)=a R^{m}$ and have discussed several solutions corresponding to different values of the exponential parameter. In the second scheme, for the specific choice of the shape functions we have reconstructed $f(R)$. We also check the energy conditions for both schemes.

Our plan of work is as follows: In Sec.II, we write down the field equations of a Morris-Thorne wormhole in $f(R)$ gravity supported by anisotropic matter. In Sec.III, we solve the field equations by assuming a power-law form: $F(R)=a R^{m}$ and discussed several solutions corresponding to different values of $\mathrm{m}$. In Sec.IV, we solve the same field equations by inserting specific forms of shape functions and have reconstructed $f(R)$ in all cases and we conclude in Sec. IV.

\footnotetext{
*Electronic address: rahaman@iucaa.ernet.in

${ }^{\dagger}$ Electronic address: ayan7575@yahoo.co.in

‡Electronic address: mjamil@camp.nust.edu.pk

§lectronic address: abanilyadav@yahoo.co.in

『Electronic address: humaira.idris@gmail.com
} 


\section{FIELD EQUATIONS IN $F(R)$ GRAVITY}

The metric describing a static spherically symmetric wormhole spacetime is given by

$$
d s^{2}=-e^{2 \Phi(r)} d t^{2}+\frac{d r^{2}}{1-\frac{b(r)}{r}}+r^{2}\left(d \theta^{2}+\sin ^{2} \theta d \phi^{2}\right) .
$$

Here, $\Phi(r)$ is a gravitational redshift function and $b(r)$ is the shape function. The radial co-ordinate $\mathrm{r}$, decreases from infinity to a minimum value $r_{0}$, and then increases from $r_{0}$ to infinity. The minimum value of $r_{0}$, represents the location of the wormhole throat where $b\left(r_{0}\right)=r_{0}$, satisfying the flaringout condition $b-b^{\prime} r / b^{2}>0$ and $b^{\prime}\left(r_{0}\right)<1$, that are imposed to have wormhole solution.

For our wormhole in $\mathrm{F}(\mathrm{R})$ gravity, we assume that the matter content of the wormhole is anisotropic fluid source whose energy-momentum tensor is given by []

$$
T_{\nu}^{\mu}=\left(\rho+p_{r}\right) u^{\mu} u_{\nu}-p_{r} g_{\nu}^{\mu}+\left(p_{t}-p_{r}\right) \eta^{\mu} \eta_{\nu}
$$

with $u^{\mu} u_{\mu}=-\eta^{\mu} \eta_{\mu}=1$, and $u^{\mu} \eta_{\mu}=0$. Here the vector $u^{\mu}$ is the fluid 4-velocity and $\eta^{\mu}$ is a space-like vector orthogonal to $u^{\mu}$.

Following Lobo et al's [1], we have the following gravitational field equations in $f(R)$ gravity as

$$
\begin{aligned}
\rho(r) & =\frac{F b^{\prime}}{r^{2}} \\
p_{r}(r) & =-\frac{F b}{r^{3}}+\frac{F^{\prime}}{2 r^{2}}\left(b^{\prime} r-b\right)-F^{\prime \prime}\left(1-\frac{b}{r}\right), \\
p_{t}(r) & =-\frac{F^{\prime}}{r}\left(1-\frac{b}{r}\right)-\frac{F}{2 r^{3}}\left(b^{\prime} r-b\right) .
\end{aligned}
$$

Here, prime indicate derivative with respect to $\mathrm{r}$ and $\rho, p_{r}$ and $p_{t}$ are the energy density, radial pressure and tangential pressure, respectively. These equations are the generic expressions of the matter threading wormhole with constant redshift function which simplifies the calculations of field equations, and provide interesting exact wormhole solutions, where $F=\frac{d f}{d R}$ and the curvature scalar, $R$, is given by

$$
R(r)=2 \frac{b^{\prime}(r)}{r^{2}}
$$

\section{WORMHOLES FOR A GIVEN $F(R)$ FUNCTION}

We take a power-law form

$$
F(R)=a R^{m}
$$

Here, $a$ is a constant and $\mathrm{m}$ is an integer.

We are trying to solve the field equations given above in non-commutative geometry with Lorentzian distribution. Here we take the energy density of the static and spherically symmetric smeared and particle-like gravitational source of the following [12]

$$
\rho=\frac{M \sqrt{\phi}}{\pi^{2}\left(r^{2}+\phi\right)^{2}} .
$$

Here, the mass $M$ could be a diffused centralized object such as a wormhole and $\theta$ is noncommutative parameter.

Using Eq.(6) in Eq. (7), gives

$$
F(R)=a\left(\frac{2 b^{\prime}}{r^{2}}\right)^{m}
$$

Substituting Eqs. (8) and (9) in Eq. (3), we obtain the shape function given by

$$
b(r)=\int r^{2}\left[\frac{M \sqrt{\phi}}{2^{m} \pi^{2} a\left(r^{2}+\phi\right)^{2}}\right]^{\frac{1}{1+m}}+C
$$

where $C$ is a constant of integration.

To get the exact physical characteristics, we discuss several models resulting for different choices of $\mathrm{m}$. 

A. $m=0$

From Eq.(10), the assumption $\mathrm{m}=0$, gives the shape function of the form

$$
b(r)=\frac{M \sqrt{\phi}}{2 a \pi^{2}}\left[\frac{\arctan \left(\frac{r}{\sqrt{\phi}}\right)}{\sqrt{\phi}}-\frac{r}{r^{2}+\phi}\right]+C .
$$

By putting (11) in (6), we get

$$
R(r)=\frac{2 M \sqrt{\phi}}{\pi^{2} a\left(r^{2}+\phi\right)^{2}}
$$

From Eqs. (4) and (5), using the shape function in Eq.(11) the expressions for pressures (radial and tangential) become

$$
\begin{gathered}
P_{r}(r)=\frac{a}{r^{3}}\left[\frac{M \sqrt{\phi}}{2 a \pi^{2}}\left(\frac{r}{r^{2}+\phi}-\frac{1}{\sqrt{\phi}} \arctan \left(\frac{r}{\sqrt{\phi}}\right)\right)-C\right] \\
P_{t}(r)=\frac{M \sqrt{\phi}}{4 \pi^{2} r^{3}}\left[\frac{1}{\sqrt{\phi}} \arctan \left(\frac{r}{\sqrt{\phi}}\right)-\frac{r}{\left(r^{2}+\phi\right)}-\frac{2 r^{3}}{\left(r^{2}+\phi\right)^{2}}+\frac{2 a C \pi^{2}}{M \sqrt{\phi}}\right] .
\end{gathered}
$$

We consider that the Lorentzian distribution of particle-like gravitational source given in Eq.(8), which is positive for the noncommutative parameter $\theta>0$. The shape function given in Eq.(11) is asymptotically flat because of $b(r) / r \rightarrow 0$ as $r \rightarrow \infty$ and the redshift function is constant everywhere. In fig.(1), corresponding to $\mathrm{m}=0$, the throat of the wormhole is located at $\mathrm{r}=1.5$, where $\mathcal{G}(r)=\mathrm{b}(\mathrm{r})$-r cuts the $\mathrm{r}$-axis ( upper right in Fig.(1)) and also $\mathcal{G}(r)<0$, i.e., $\mathrm{b}(\mathrm{r})-\mathrm{r}<0$, which implies that $\mathrm{b}(\mathrm{r})<\mathrm{r}$ for $\mathrm{r}>r_{0}$, satisfy the fundamental property of shape function which indicates in Fig.(1) (upper left). From the Fig.(1), it is also clear that, for $\mathrm{r}>r_{0}, \mathcal{G}(r)$ is decreasing function, therefore $\mathcal{G}^{\prime}(r)<0$ and correspondingly $b^{\prime}\left(r_{0}\right)<1$, which satisfies the flare-out condition.

According to a Fig.(1) (left and right, middle position) the radial pressure $\left(p_{r}\right)$ is negative and transverse pressure $\left(p_{t}\right)$ is positive outside the throat of the wormhole for the choice of the parameters $\mathrm{M}=10, \mathrm{C}=1, \mathrm{a}=1$ and $\phi=$ 1 , respectively, which shows that the wormholes violates the null energy condition (NEC) as well as weak energy conditions (WEC).

\section{B. $m=1$}

Similarly, from Eq. (10), we get the shape function for $\mathrm{m}=1$

$$
b(r)=\sqrt{\frac{M \sqrt{\phi}}{2 a \pi^{2}}}\left[r-\sqrt{\phi} \arctan \left(\frac{r}{\sqrt{\phi}}\right)\right]+C .
$$

The corresponding value of Ricci scalar becomes

$$
R(r)=\sqrt{\frac{2 M \sqrt{\phi}}{a \pi^{2}\left(r^{2}+\phi\right)^{2}}} .
$$

Moreover, the radial and transverse pressures turn out as

$$
\begin{aligned}
P_{r}(r)= & \sqrt{\frac{2 M a \sqrt{\phi}}{\pi^{2}}}\left[\frac{1}{r^{3}\left(r^{2}+\phi\right)}\left[\sqrt{\frac{M \sqrt{\phi}}{2 a \pi^{2}}}\left(\sqrt{\phi} \arctan \left(\frac{r}{\sqrt{\phi}}\right)-r\right)-C\right]\right. \\
& -\frac{1}{r\left(r^{2}+\phi\right)^{2}}\left[\sqrt{\frac{M \sqrt{\phi}}{2 a \pi^{2}}} \frac{r^{3}}{\left(r^{2}+\phi\right)}-\sqrt{\frac{M \sqrt{\phi}}{2 a \pi^{2}}}\left(r-\sqrt{\phi} \arctan \left(\frac{r}{\sqrt{\phi}}\right)\right)-C\right] \\
& \left.-\frac{\left(6 r^{4}+4 r^{2} \phi-2 \phi^{2}\right)}{\left(r^{2}+\phi\right)^{4}}\left[1-\sqrt{\frac{M \sqrt{\phi}}{2 a \pi^{2}}}\left(1-\frac{\sqrt{\phi}}{r} \arctan \left(\frac{r}{\sqrt{\phi}}\right)\right)-\frac{C}{r}\right]\right] .
\end{aligned}
$$



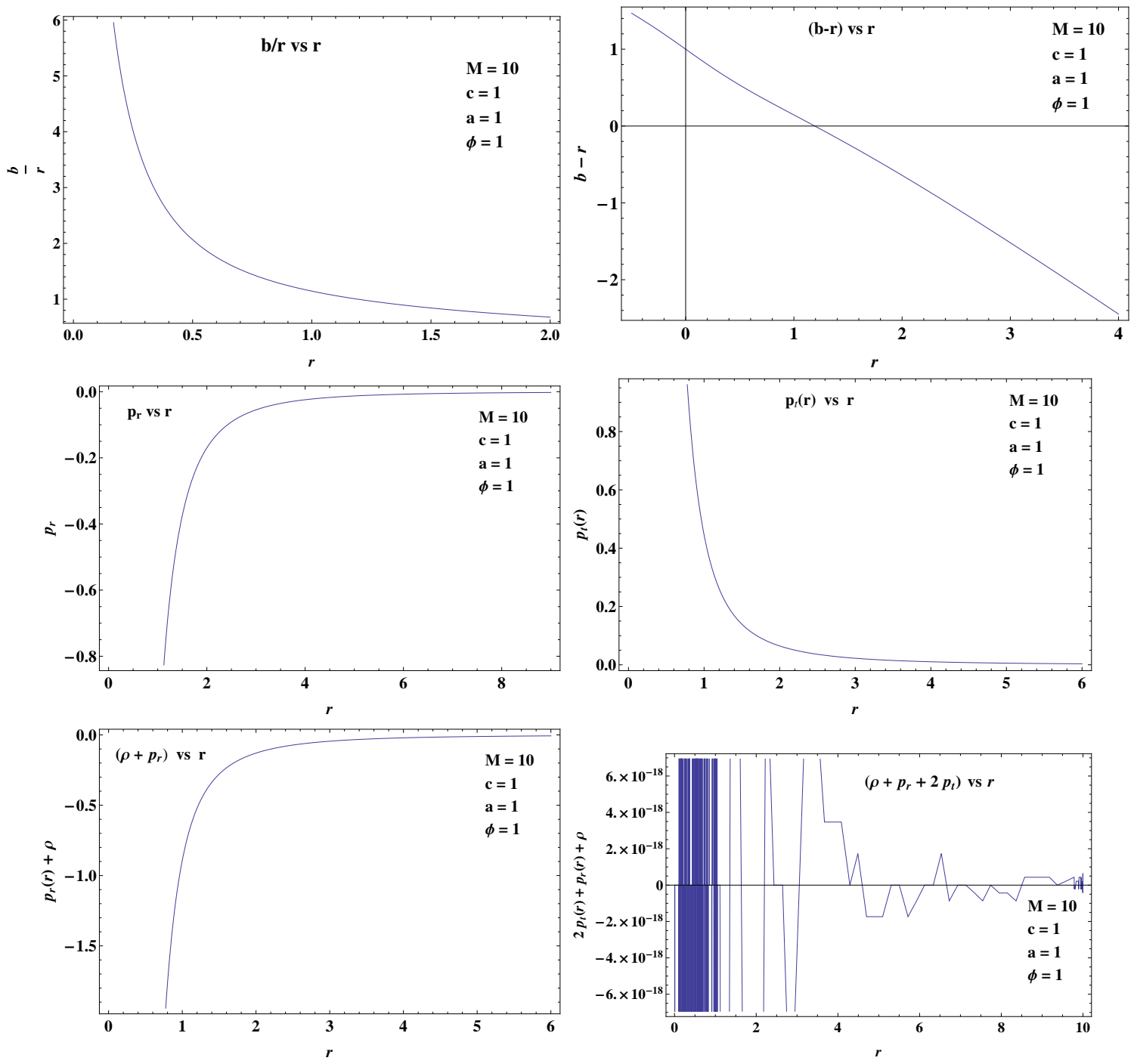

FIG. 1: Graphs for the case $m=0$.

$$
\begin{aligned}
P_{t}(r)= & \sqrt{\frac{2 M a \sqrt{\phi}}{\pi^{2}}}\left[\frac{2}{\left(r^{2}+\phi\right)^{2}}\left[1-\sqrt{\frac{M \sqrt{\phi}}{2 a \pi^{2}}}\left(1-\frac{\sqrt{\phi}}{r} \arctan \left(\frac{r}{\sqrt{\phi}}\right)\right)-\frac{C}{r}\right]\right. \\
& \left.-\frac{1}{2 r^{3}\left(r^{2}+\phi\right)}\left[\sqrt{\frac{M \sqrt{\phi}}{2 a \pi^{2}}} \frac{r^{3}}{\left(r^{2}+\phi\right)}-\sqrt{\frac{M \sqrt{\phi}}{2 a \pi^{2}}}\left(r-\sqrt{\phi} \arctan \left(\frac{r}{\sqrt{\phi}}\right)\right)-C\right]\right] .
\end{aligned}
$$

In Fig.(2), the graphs are drawn for the choice of the same parameters given in Fig.(1), corresponding to $\mathrm{m}=1$. The throat of the wormhole is located at $\mathrm{r} \sim 1.5$, where $\mathcal{G}$ cuts $\mathrm{r}$-axis and the shape function satisfy all the necessary conditions of as shown in the Figs.(2).

Here also from Fig.(2), (lower left) the Null Energy Condition (NEC) and the Weak Energy Condition (WEC) are violated, conditions that are necessary to hold a wormhole open. It is interesting to note that the Strong Energy Condition (SEC) is satisfied which shown in Fig(2), (lower right).

\section{WORMHOLE SOLUTION FOR A GIVEN SHAPE FUNCTION}

We developed the literature by considering several interesting shape function. 

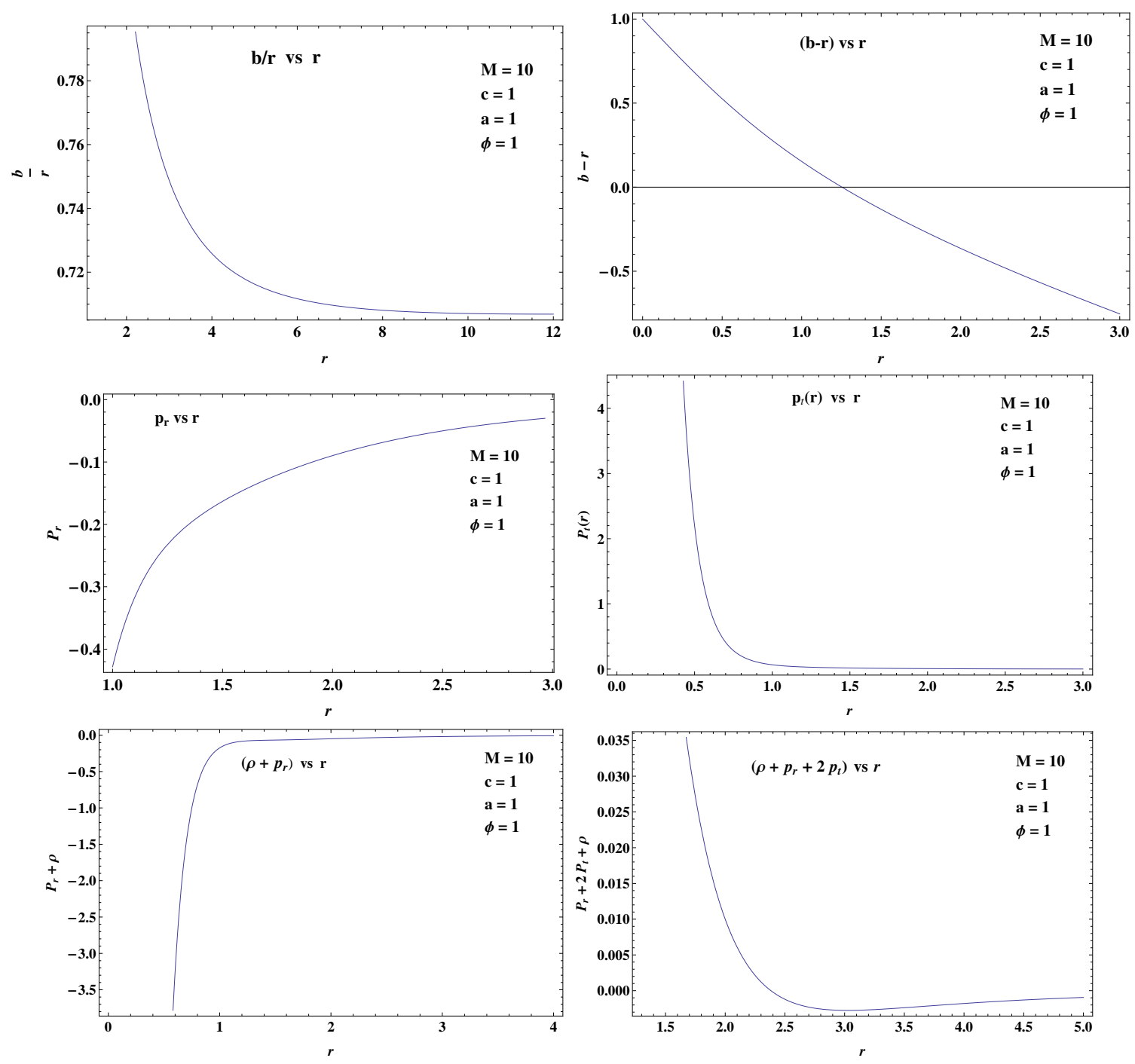

FIG. 2: Plots for case $m=1$.

A. $b(r)=r_{0}\left(\frac{r}{r_{0}}\right)^{\alpha}$

Here, we consider a shape function of the form

$$
b(r)=r_{0}\left(\frac{r}{r_{0}}\right)^{\alpha}
$$

which satisfy the flare-out conditions for $b^{\prime}\left(r_{0}\right)=\alpha<1$, and that for $\mathrm{r} \rightarrow \infty$ we have $\mathrm{b}(\mathrm{r}) / \mathrm{r}=\left(r_{0} / r\right)^{1-\alpha} \rightarrow 0$.

Putting Eq. (19) in (6), we have

$$
R(r)=\frac{2 \alpha}{r^{2}}\left(\frac{r}{r_{0}}\right)^{\alpha-1}
$$

and, Substituting Eqs. (8) and (19) in (3), we get

$$
F(r)=\frac{M \sqrt{\phi}}{\alpha \pi^{2}}\left(\frac{r_{0}}{r}\right)^{\alpha-1}\left[\frac{r^{2}}{\left(r^{2}+\phi\right)^{2}}\right] .
$$

Using gravitational field equations (3)-(5), the components of radial and transverse pressure, given by the following 
form

$$
\begin{aligned}
p_{r}(r)= & \frac{M \sqrt{\phi}}{\alpha \pi^{2}\left(r^{2}+\phi\right)^{2}}\left[-1+\frac{(\alpha-1)}{2}\left\{(3-\alpha)-\frac{4 r^{2}}{r^{2}+\phi}\right\}+\left(1-\left(\frac{r}{r_{0}}\right)^{1-\alpha}\right)\{(3-\alpha)(2-\alpha)\right. \\
& \left.\left.+\frac{4(2 \alpha-5) r^{2}}{\left(r^{2}+\phi\right)}+\frac{8 r\left(2 r^{3}-\phi\right)}{\left(r^{2}+\phi\right)^{2}}\right\}\right], \\
p_{t}(r)= & \frac{M \sqrt{\phi}}{\alpha \pi^{2}\left(r^{2}+\phi\right)^{2}}\left[\frac{(1-\alpha)}{2}+\left(1-\left(\frac{r}{r_{0}}\right)^{1-\alpha}\right)\left\{(3-\alpha)-\frac{4 r^{2}}{r^{2}+\phi}\right\}\right] .
\end{aligned}
$$

In Fig.(3), the graphs are drawn for the choice of the parameters $\mathrm{M}=10, r_{0}=1, \alpha=-1$ and $\phi=1$, respectively. Here also the throat of the wormhole is located at $\mathrm{r} \sim 1.5$, where $\mathcal{G}$ cuts $\mathrm{r}$-axis shown in the Fig.(3), (upper right) and the energy conditions are same as in previous cases shown in left and right lower Fig.(3).
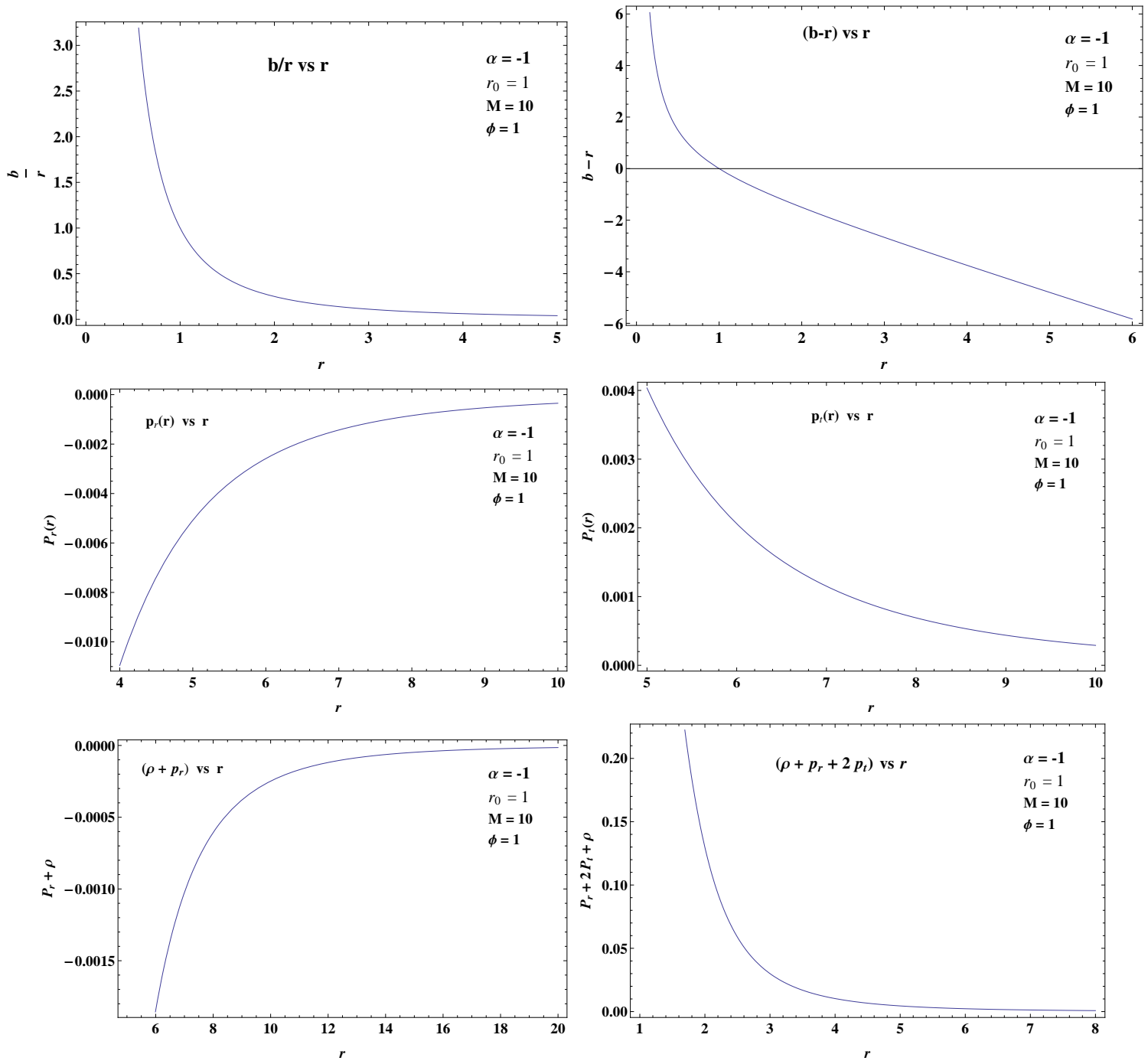

FIG. 3: Plots for the case A of Section IV. 
B. A particular shape function : $b(r)=\operatorname{Atan}^{-1} C r$

Let us consider a shape function of the form

$$
b(r)=\operatorname{Atan}^{-1} C r,
$$

so that $\mathrm{b}(\mathrm{r}) / \mathrm{r}=\operatorname{Atan}^{-1} \mathrm{Cr} / \mathrm{r} \rightarrow 0$ for $\mathrm{r} \rightarrow \infty$ (by L'Hospital's rule), which met the asymptotically flat conditions. However, one may also verify that $b^{\prime}\left(r_{0}\right)=A C /\left(1+C^{2} r_{0}^{2}\right)<1$ i.e., the fundamental property of a wormhole is that a flaring out condition at the throat is met for the choice of the parameters.

using Eq. (24) in (6), we have

$$
R(r)=\frac{2}{r^{2}}\left(\frac{A C}{1+C^{2} r^{2}}\right)
$$

The form of $F(r)$ function becomes

$$
F(r)=\frac{M \sqrt{\phi}}{\pi^{2} A C} \frac{r^{2}\left(1+C^{2} r^{2}\right)}{\left(r^{2}+\phi\right)^{2}} .
$$

The components of radial and transverse pressure become

$$
\begin{aligned}
P_{r}(r)= & -\frac{M \sqrt{\phi}}{\pi^{2} A C}\left[\frac{A}{r} \tan ^{-1} C r \frac{\left(1+C^{2} r^{2}\right)}{\left(r^{2}+\phi\right)^{2}}-\left(\frac{A C r}{1+C^{2} r^{2}}-\operatorname{Atan}^{-1} C r\right)\left(\frac{1+2 C^{2} r^{2}}{r\left(r^{2}+\phi\right)^{2}}-\frac{2\left(r+C^{2} r^{3}\right)}{\left(r^{2}+\phi\right)^{3}}\right)\right. \\
& \left.+\left(1-\frac{A}{r} \tan ^{-1} C r\right)\left(\frac{2+12 C^{2} r^{2}}{\left(r^{2}+\phi\right)^{2}}-\frac{4\left(5 r^{2}+9 C^{2} r^{4}\right)}{\left(r^{2}+\phi\right)^{3}}+\frac{24 r\left(r^{3}+C^{2} r^{5}\right)}{\left(r^{2}+\phi\right)^{4}}\right)\right], \\
P_{t}(r)= & -\frac{M \sqrt{\phi}}{\pi^{2} A C}\left[\left(1-\frac{A}{r} \tan ^{-1} C r\right)\left(\frac{2+4 C^{2} r^{2}}{\left(r^{2}+\phi\right)^{2}}-\frac{4\left(r^{2}+C^{2} r^{4}\right)}{\left(r^{2}+\phi\right)^{3}}\right)\right. \\
& \left.+\left(\frac{A C}{1+C^{2} r^{2}}-\frac{A}{r} \tan ^{-1} C r\right)\left(\frac{1+C^{2} r^{2}}{2\left(r^{2}+\phi\right)^{2}}\right)\right] .
\end{aligned}
$$

In Fig.(4), the graphs are drawn for the choice of the parameters $M=10, a=1, A=2, C=1$ and $\phi=1$, respectively. Here also the throat of the wormhole is located at $\mathrm{r} \sim 2$, where $\mathcal{G}$ cuts $\mathrm{r}$-axis shown in the Fig.(4), (upper right) and the energy conditions are same as in previous cases shown in left and right lower portion of Fig.(4). 

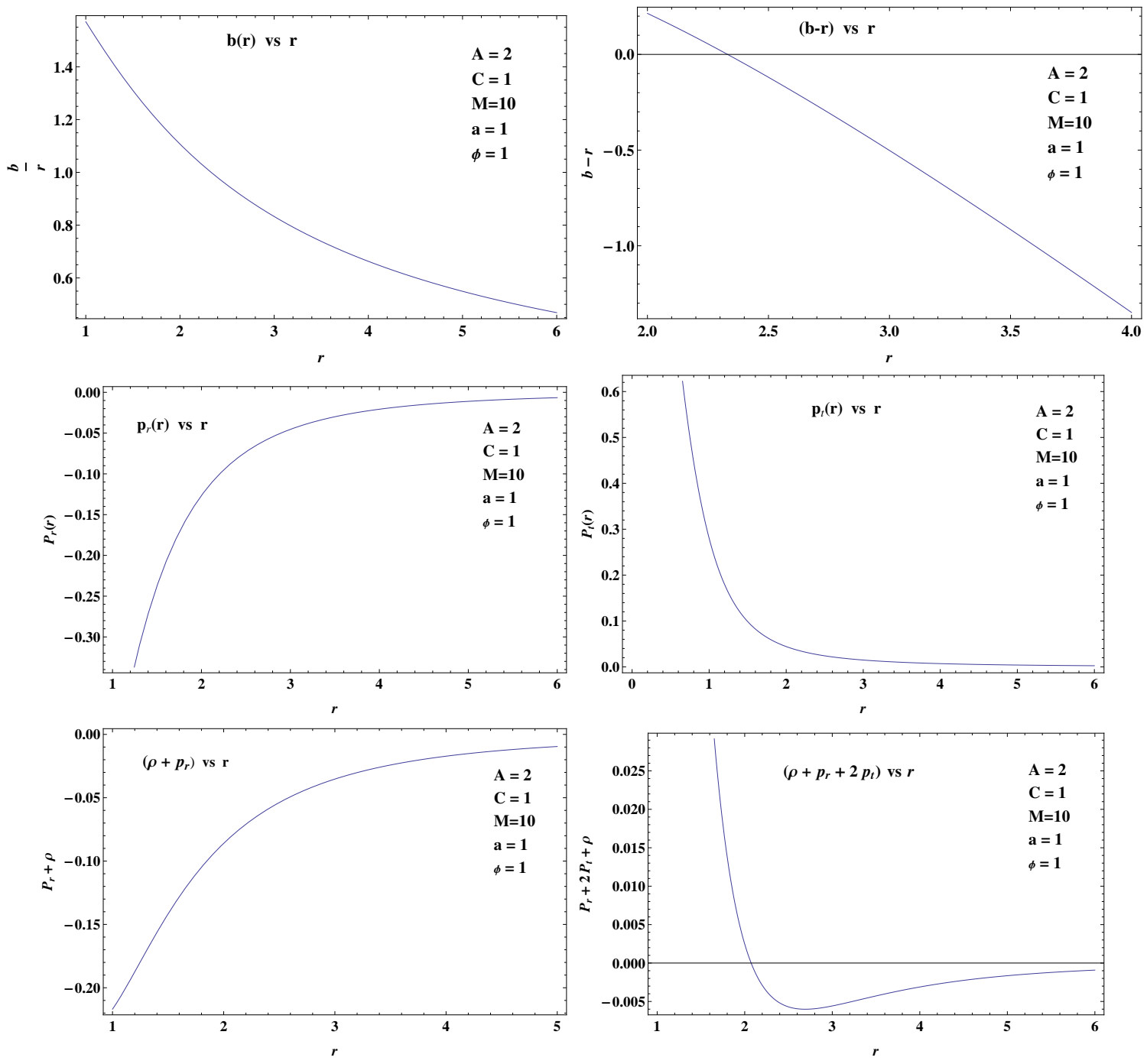

FIG. 4: Plots of case B of Section IV.

\section{CONCLUDING REMARKS}

In this paper, we derived some new exact solutions of static wormholes in $f(R)$ gravity supported by the matter possesses Lorentizian density distribution of a particle-like gravitational source. We derive the wormhole's solutions in two possible schemes for a given Lorentzian distribution. The first model assumes the power-law form $F(R)$ whereas the second model discussed assumes a particular shape function which allows the reconstruction of $f(R)$. For the power-law form of $F(R)$ with $m=1$ is interesting as in this case the null energy condition is violated, but the strong energy condition is met. For the second model, we have considered two particular shape functions and have reconstructed $f(R)$ in both cases. In these two cases, the null energy condition are once again violated, but the strong energy conditions are met. All the solutions assume zero tidal forces which automatically that the wormholes are traversable [11]. 


\section{Acknowledgments}

FR is thankful to the authority of Inter-University Centre for Astronomy and Astrophysics, Pune, India for providing them Visiting Associateship under which a part of this work was carried out. AB is also thankful to IUCAA for giving him an opportunity to visit IUCAA where a part of this work was carried out. FR is also thankful UGC, Govt. of India under research award scheme, for providing financial support.

[1] E. Teo, Phys. Rev. D 58 (1998) 024014; P.K.F. Kuhfittig, Phys. Rev. D 67 (2003) 064015; P.E. Kashargin and S.V. Sushkov, arXiv:0710.5656 [gr-qc]; Mubasher Jamil, Peter K.F. Kuhfittig, Farook Rahaman, Sk. A Rakib, Eur. Phys. J. C 67 (2010) 513-520

[2] G. Clement, Phys. Rev. D 51 (1995) 6803

[3] J.P.S. Lemos et al, Phys. Rev. D 68 (2003) 064004; F Rahaman et al, Gen.Rel.Grav. 39 (2007) 145

[4] J.P.S. Lemos and F.S.N. Lobo, Phys. Rev. D 78 (2008) 044030; F Rahaman et al, Gen.Rel.Grav. 38 (2006) 1687 ; F Rahaman et al, Mod.Phys.Lett. A24 (2009) 53 ; F Rahaman et al, Class.Quant.Grav. 28 (2011) 155021 ; F Rahaman et al, Int.J.Theor.Phys. 51 (2012) 1680.

[5] S-Won Kim and H. Lee, Phys. Rev. D 63 (2001) 064014; F Rahaman et al, Int.J.Theor.Phys. 48 (2009) 1637

[6] F.S.N. Lobo, arXiv:0710.4474 [gr-qc]

[7] M.S. Morris and K.S. Thorne, Am. J. Phys. 56 (1988) 395; M.S. Morris et al, Phys. Rev. Lett. 61 (1988) 1446

[8] T.A. Roman, Phys. Rev. D 47 (1993) 1370

[9] S.W. Hawking, Phys. Rev. D 46 (1992) 603

[10] M. S. Morris and K. S. Thorne, Am. J. Phys. 56, 395 (1988); M. Visser, Loretzian wormholes: from Einstein to Hawking AIP Press (1995).

[11] F. S. N. Lobo, M. A. Oliveira, Phys. Rev. D 80, 104012 (2009).

[12] S. Hamid Mehdipour, Eur. Phys. J. Plus (2012) 127: 80 